\title{
When science meets Google: Reflections on research and evidence in the age of science deniers
}

Noni E. MacDonald, MD, MSc, FRCP

Faculty of Medicine, Dalhousie University and Department of Pediatrics, IWK Health Centre, Halifax, NS

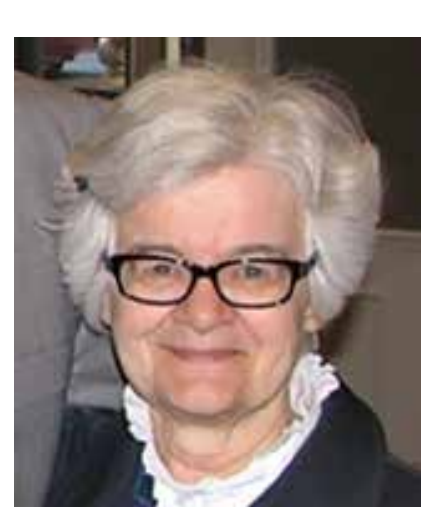

\section{$\mathrm{BIO}$}

Noni is a former Dean of Medicine at Dalhousie University. Her two current major areas of interest are 1) Vaccines, including vaccine safety, hesitancy, demand, pain mitigation, education and policy especially through her work with the World Health Organization as a member of Strategic Advisory Group of Experts on Immunization and 2) MicroResearch, building capacity in community-focused research in developing countries and now also in Canada to help interdisciplinary health professionals find local solutions for community health problems that fit the context, culture and resources. Noni has long been internationally recognized as an advocate for children and youth health and as a leader in pediatric infectious disease and global health.

$\mathrm{M}$ uch of my research career has centered on vaccines-trying to find answers to questions that arose at the bedside, in the clinic and in immunization program delivery. Projects have travelled across the full spectrum from basic science bench studies, to clinical trials, to behavioural research questions, to knowledge translation and implementation studies with vaccine education, advocacy and policy work thrown into the mix. Vaccinology has indeed been one of my life passions. Having worked in this area for over three decades now, I well recognize that vaccine science deniers are not new. They have been arguing against immunization since Jenner pushed forward small pox vaccination in the late $1700 \mathrm{~s}$. What is new are their growing prominence and much wider reach. They have stirred up concerns about vaccines, leading to hesitancy and decreased acceptance of vaccines. Vaccine hesitancy is now part of the discourse on vaccine acceptance where this was little talked about a decade ago. Vaccine hesitancy is now well recognized as a serious, albeit complex, problem that affects almost every country in the world, and concerns about vaccine risk and benefits (the science of vaccines) are now the leading reported reason globally for vaccine hesitancy ${ }^{1}$.

\section{Why is vaccine hesitancy so prominent now?}

To understand vaccine hesitancy we need to review how risk is perceived and decisions about vaccine acceptance are made. While we live in the midst of a tsunami of information, some is evidence based but much not. We as individuals, even those with good science backgrounds, make decisions based upon our core beliefs $^{2,3}$. We screen incoming information. If it agrees with our beliefs, it is well heard; if not, it is rejected-a term called assimilation bias (Figure 1). Our beliefs influence our vaccine acceptance decisions, and rationale review of the evidence is rarely part of this decision making ${ }^{4}$. Instead, we are strongly influenced by what we think others around us are doing or are expecting us to do. We more readily assimilate anecdotes and stories than evidence. We see and hear what we believe rather than believe what we see and hear. Vocal vaccine deniers play to our emotions. Fact-based reality is less important to those who are being made anxious about immunization than telling them what they want to hear in a way that 


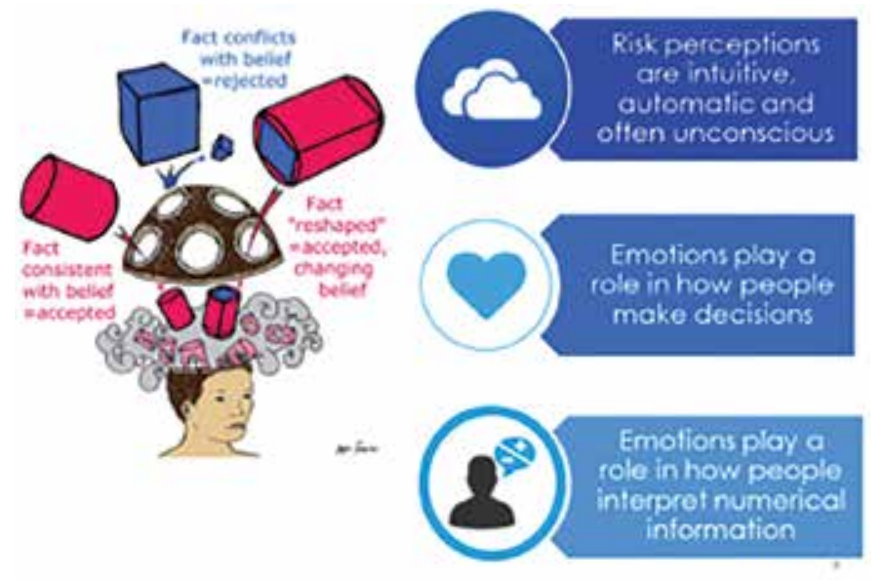

Figure 1. Risk perception, assimilation bias and vaccine acceptance Copyright N.E. MacDonald; used with permission by CIM.

This is not new. We have made decisions based on our beliefs for millennia but what is new is how easily vocal vaccine science deniers can now get their messages out far and wide. The web means anyone and everyone can call themselves an expert. With the rights skills and tactics, these pseudo experts can influence thousands and thousands instead of only a few close neighbours ${ }^{5}$. To add to this, negative vaccine beliefs are socially contagious, spreading in a fashion similar to a microbial outbreak ${ }^{6}$.

\section{What can we do? What are evidence-based strategies to address the social contagion aspect of vaccine science deniers?}

Internet search engines like Google $\mathrm{e}^{\mathrm{TM}}$ and the hundreds of social networking sites like Facebook ${ }^{\mathrm{TM}}$ and Twitter${ }^{\mathrm{TM}}$ are not going to disappear in the near future and more will be developed. Policing them so that only evidence-based data and comments are allowed is neither possible, nor practical nor ethical. However, there is a growing body of evidence that suggests the public can be influenced by evidence, and that this evidence can change their beliefs. They can be inoculated against science deniers and fake news if the right tactics are used.

Presenting the evidence is not enough. The problem is not just a knowledge gap. Research on countering vaccine science deniers has found that belief in a scientific fact increases as scientific consensus is highlighted and emphasized ${ }^{7}$. In vaccinology, that means health care pro- fessionals and scientists coming together to emphasize that vaccines are safe and effective and serious diseases can occur if a person is not immunized. The European Region of the World Health Organization has developed evidence-informed guidance on how to address vocal vaccine deniers in a public forum (http://www. euro.who.int/_data/assets/pdf_file/0005/315761/ Best-practice-guidance-respond-vocal-vaccine-deniers-public.pdf?ua=1).

The document highlights the topics deniers focus on and the typical tactics that are used ${ }^{8}$. The target for the health care professional in the public forum is not the vocal vaccine science denier but rather the audience who is listening. The anti-vaccine tactics used by vocal vaccine deniers are similar to those used by fake news promulgators and by science deniers in other fields: invoking fake experts and conspiracies; selective use of evidence; promotion of impossible expectations (100\% safe and effective); misrepresentation of evidence; manufacturing of doubt; and use of logic fallacies such as "after it therefore because of it". The task of repudiating the denier's miss-information in a public forum is neither simple nor easy, but it can be done. Building of trust, an amalgam of demonstrating both caring and competen$c y$, between the pro-scientific evidence speaker and the listening audience, is an important element. Unmasking the science deniers' tactics helps the audience see the denier's information in a different light. No one likes to be conned. Repudiation must be done respectfully and carefully or the pro-scientific evidence speaker may push the audience to develop empathy for the science denier and then the audience may not be able to hear the evidence. Although more work is needed, attitudinal resistance to science deniers' arguments can be grown in the general public by pre-emptively highlighting false claims and refuting potential counterarguments ${ }^{9}$. Building on this concept of protective inoculation, the van der Linden group at Cambridge University are testing a game app entitled "Bad News". Players compete to become "a disinformation and fake news tycoon" (https:// www.getbadnews.com/\#intro). By practising the fake news tactics noted above, the participant should then more quickly recognize situations in which they are being applied and be more immune to the fake messages. As a believer in the value and importance of vaccines, I 
have hope that this evidence-based game strategy just might work to stem the impact of fake news, fake science and science deniers' messages-or maybe this is just a fake science belief.

\section{What have I learned as I have reflected on vocal science deniers?}

\section{One's own beliefs shape what one sees and} hears. I am now more aware of how easily and often we are blind to new evidence if the findings contradict our beliefs. I think about this when I review a paper or a grant and I feel my hackles going up because a "sacred cow" is being gored. I now sit back, ponder the evidence and try to keep a more open mind.

2. Interdisciplinarity enhances research. I now eagerly welcome different perspectives and backgrounds on research teams working on complex problems like vaccine hesitancy. The insights and contributions of medical anthropologists, psychologists, sociologists, ethicists, communication experts, marketing experts and lawyers, as well as members of the communities being studied, are so valuable.

3. Leaving your comfort zone can invigorate your research.

4. Addressing vaccine hesitancy is complex, hard work. Working with vaccine hesitant or sciencedenying parents, patients, health care professionals, colleagues or members of the public is not for the faint of heart but there are evidence-based strategies that can help

5. Communication needs to fit the target to be heard. The mantra to address vaccine hesitancy is to communicate, communicate and communicate some more. While this is evidence-based and "true", this now has new meaning-we must package our evidence so it can be heard.

6. Fake news and science deniers are not going away. The problem of fake news extollers and science deniers is not going away, but by learning about their tactics and recognizing what they are doing we can help protect ourselves, our families, our clinical and research colleagues, our patients and the public. While the genie can never be put back into the lamp because of the internet, we may be able to defang this evil dragon through protective information inoculation.
In closing, I wonder if I had understood 20 or 30 years ago how beliefs shape what we see, hear and decide, if this would have altered the design and/or knowledge translation plans for many of my earlier projects and even my career path. I think back to how often over the years I have been frustrated because policy makers, hospital administrators and fellow health care workers could not "hear" the evidence and see its implications when a "sacred" untouchable program was shown to be flawed. How many times did I too reject evidence because I could not hear it? I hope I am better able to hear and see now.

\section{REFERENCES}

1. Lane S, MacDonald NE, Marti M, Dumolard L. Vaccine hesitancy around the globe: analysis of three years of WHO/UNICEF Joint Reporting Form data-2015-2017. Vaccine. 2018; 36(26):3861-7.

2. Smith JC, Appleton M, MacDonald NE. Building confidence in vaccines. Adv Exp Med Biol. 2013; 764: 81-98.

3. Kahan DM. A Risky Science Communication Environment for Vaccines. Science. 2013; 342(6154):53-4.

4. Dube E, MacDonald NE. Managing the risks of vaccine hesitancy and refusals. Lancet Infectious Diseases. 2016; 16: 518-9.

5. Kata A. Anti-vaccine activists, Web 2.0, and the postmodern paradigm - An overview of tactics and tropes used online by the anti-vaccination movement. Vaccine. 2012; 30: 3778- 89.

6. Bauch CT, Galvani AP. Social Factors in Epidemiology. Science. 2013; 342 (6154): 47-9.

7. van der Linden SL, Clarke CE, Maibach EW. Highlighting consensus among medical scientists increases public support for vaccines: evidence from a randomized experiment. BMC Public Health. 2015; 15:1207.

8. Schmid P, MacDonald NE, Habersaat K, Butler R. Commentary to: How to respond to vocal vaccine deniers in public. Vaccine. 2018; 36: 196-8.

9. van der Linden, S Leiserowitz A, Rosenthal S, Maibach E. Inoculating the Public against Misinformation about Climate Change. Science. 2017; 358(6367):1141-2.

\section{CORRESPONDENCE TO:}

E-mail noni.macdonald@dal.ca 